\title{
Propagation Measurements and Estimation of Channel Propagation Models in Urban Environment
}

\author{
*Yahia Zakaria ${ }^{1,2,3}$, Lubomir Ivanek ${ }^{1}$, Ivan Glesk ${ }^{3}$ \\ ${ }^{1}$ Department of Electrical Engineering, Faculty of Electrical Engineering and Computer Science, VSB-Technical \\ University of Ostrava, 17. listopadu 15, 70833 Ostrava, Czech Republic \\ [e-mail: yahia.salem.st@vsb.cz, lubomir.ivanek@vsb.cz] \\ ${ }^{2}$ Engineering Research Division, National Research Centre (NRC), Dokki, P.O. 12622, Cairo, Egypt \\ [e-mail: yahia.zk82@yahoo.com] \\ ${ }^{3}$ Deparment of Electronic and Electrical Engineering, University of Strathclyde, 204 George Street, Glasgow G1 \\ 1XW, United Kingdom, UK \\ [e-mail: yahia.salem.2016@uni.strath.ac.uk, ivan.glesk@strath.ac.uk] \\ *Corresponding author: Yahia Zakaria
}

Received August 20, 2016; revised January 9, 2017; accepted February 3, 2017;

published May 31, 2017

\begin{abstract}
Wireless communication is a telecommunication technology, which enables wireless transmission between the portable devices to provide wireless access in all types of environments. In this research, the measurements and various empirical models are analysed and compared in order to find out a suitable propagation model to provide guidelines for cell planning of wireless communication systems. The measured data was taken in urban region with low vegetation and some trees at $900 \mathrm{MHz}$ frequency band. Path loss models are useful planning tools, which permit the designers of cellular communication to obtain optimal levels for the base station deployment and meeting the expected service level requirements. Outcomes show that these empirical models tend to overestimate the propagation loss. As one of the key outputs, it was observed that the calculations of Weissberger model fit with the measured data in urban environment.
\end{abstract}

Keywords: Propagation, Urban, Weissberger's Model, COST 231 Walfish-Ikegami (W-I) Model 


\section{Introduction}

In wireless communication system, the losses that occurred between transmitter and receiver is known as propagation path loss. Path loss is a main factor in the analysis and design of cellular communication system. Furthermore, the electromagnetic waves usually cannot directly arrive the receiver due to many obstacles that prevent the line of sight path. Thus, propagation analysis is very important in evaluating the signal characteristics. Therefore, it is very important to have the knowledge about the electromagnetic environment where the system is operated, and the location of the transmitter and receiver. Moreover, the travelled signal from transmitter to receiver over a lot of reflection path is called multipath propagation which always causes fluctuations in the receiver signal's phase and amplitude, the mechanisms that impact the signal propagation are as follows [1]:

- Doppler Effect

- Scattering

- Reflection

- Diffraction

It is very important to gather knowledge about the type of environment of the electromagnetic wave and the position of the transmitter and receiver:

The path loss estimation may vary from one propagation model to another one due to the differences in city structures, local terrain profiles and weather. The terrain has a significant effect on the signal through its propagation from the transmitter to receiver. Obviously, hills that obstruct the path will attenuate the signal and often making reception of the signal impossible. Moreover, at low frequencies on the long wave band, it is found that signals travel best over more conductive terrain such as sea paths and areas that are damp. Dry and sandy terrain gives higher levels of attenuation. Also, doppler spread is considered as one of the critical parameter in the adaptation of wireless communication systems. It provides information about the fading rate of the channel. Moreover, knowledge of Doppler spread can improve detection and aid into transmission optimization in both physical layer and higher levels of the protocol stack [2].

Path loss definition can be extracted from the description of the widely used channel model which is Free Space Path Loss model. This model is used to compute the received signal strength when there

is clear Line Of Sight (LOS) path between the transmitter and the receiver. The power received in free space $\left(P_{r}\right)$ is given by the well-known Friis transmission equation:

$$
P_{r}(d)=\frac{P_{t} G_{r} \lambda^{2} G_{t}}{L(4 \pi d)^{2}}
$$

where $d$ is the distance between transmitter and receiver in meters; $G_{r}$ is the receiver antenna's gain; $G_{t}$ is the transmitter antenna's gain; $P_{t}$ is the transmitted power; $\mathrm{L}$ is the system loss factor, considered to be equal $1 ; \lambda$ is the wavelength in meters. 
In the lognormal path loss propagation model the average path loss for an arbitrary, $L_{p}(d)$, is expressed as a function of the distance $d$ by using a path loss exponent, independently of the presence of a direct LOS between the transmitter and the receiver units as, [3]:

$$
L_{p}(d) \alpha\left[\frac{d}{d_{0}}\right]^{n}
$$

where $n$ is the path loss exponent that indicates the rate at which the path loss increases with the distance; $\mathrm{d}_{0}$ is called the free-space close-in reference distance.

However, attention should be paid to the fact that measurements done in environments which sharing similar characteristics have shown that the path loss $L_{p}(d)$ is a random variable that has a lognormal distribution around a mean value $L_{p}(d)$, which expressed in $\mathrm{dB}$ as, [4]:

$$
L_{p}(d)=L_{f s}\left(d_{0}\right)+10 n \log \left[\frac{d}{d_{0}}\right]
$$

where, the first part is the path loss at the reference distance $d_{0}$, and the second part depends on the distance $d$ and the path-loss exponent $n$.

The research is arranged as follows: In section 2, the background and preliminaries are described whereas in section 3, literature review is stated. In section 4, the selected empirical models are described. In section 5 , the measurement process is described. In section 6 , the measurement results and the proposed empirical models are analysed. Finally, the conclusions follow in the last section.

\section{Background and Preliminary Research}

The transmission path between transmitter and receiver can vary from Line-Of-Sight (LOS) transmission to Non Line-Of-Sight (NLOS), which is obstructed by mountains and buildings [5]. In 1994, Honcharenko et al, investigated the reflection and transmission properties of concrete walls with periodic structures [6]. Through transmission path, the signals will be reflected and reached the receiver through different paths in terrestrial environment. Usually, received signals will be found to vary with position of the receiver. Many of the existing path loss prediction models have limitations, and by comparing them with the practical measured data, it will be easy to determine the most precise path loss prediction model for wireless communication systems [7].

In the 1960s and 1970s, the cellular concept was developed in Bell laboratories [8]. In 1983, Oeting declared that the difference in the received signal strength between the nearest and the farthest mobile user in a particular cell can be in the range of $100 \mathrm{~dB}$, which can cause saturation at the receiver of the weakest signal or an extravagant amount of neighbouring channel interferences, and to avoid this problem, the transmitted power at the base station should be put inversely to the effective distance of the mobile from the base station [9].

In 1990, Keenan et al, formulated a radio coverage prediction model based on building parameters. The parameters in the procedure of this model were derived from measurement data. The model provides a fast and simple way to predict the path loss in an indoor environment. Therefore, it was useful for the primary coverage prediction [10]. 
In 1992, Seidel et al, proposed path loss models based on the data measured at $914 \mathrm{MHz}$. The models are dependent on exponential path loss value versus distance relationship. In open buildings area, the path loss exponent $\mathrm{n}$ is close to 2 . For environments with many obstructions between the transmitter and the receiver, the path loss exponent can be much higher [11].

In 1993, Hashemi et al, developed a statistical model for indoor radio propagation dependent on the results of a lot of multipath propagation measurements in two office buildings. The simulation results of this model agreed with measurement results in the two office buildings, however, application of this model was not successful in factory area [12].

In 1996, Rizk et al, showed that the scattering by metallic cylinders is significant as the reflections and diffractions by testing the effects of lamppost and panel scattering in urban propagation environment [13].

In 1997, many investigations of over rooftop propagation models were introduced. The new improved analysis of electromagnetic wave propagation over irregular terrain was described by comparing the numerical simulations and measurement results. Moreover, the numerical simulations in this case offered solution that is more appropriate by taking into account the polarization problem [14].

In 1999, Vinko Erceg et al, introduced a statistical path loss model that derived from 1.9 $\mathrm{GHz}$ experimental data which gathered across the United States of America. The result showed that the reference path loss model was close to the calculated Free Space Path Loss [15].

In 2002, Saunders et al, stated that the propagation models which applied for macrocell wireless mobile systems have reported an error considered for the network design through the overall signal strength calculations to take account of the phenomenon of signal fading, and any shortage of the error will have an impact on performance of the wireless network. Therefore, the suitable solution of this problem is to adapt the parameters of empirical models with a certain reference to the specified environment in order to fulfill minimum error between the predicted and measured signal strength [16].

In 2012, three different scenarios were compared to see the effect of the construction material, layout and type of the building on the electric field strength. The simulation result showed that the surface was smooth due to the dominant direct field in the area that closed to the source point, and then the surface contains ripples due to the interferences between direct and reflected fields [17].

In 2013, Isabona et al, carried out an experimental measurement at $2100 \mathrm{MHz}$ band in Government Reservation Area, Benin City. By concentrating on different parameters, such as distance from base stations, it was noticed that the path loss increases with distance from the signal source due to a corresponding decrease in the field strength [18].

In 2015, Samimi and Rappaport, presented an omnidirectional spatial and temporal 3-dimensional statistical channel model for $28 \mathrm{GHz}$ dense urban NLOS environments. The presented methods and model can be used for millimeter-wave system-wide simulations [19].

\section{Empirical Propagation Models}

Channel modelling is important for characterized the impulse response and to estimate the path loss of a propagating channel. In this section we focus on Weissberger and COST 231 W-I models as they considered being more suitable in the area which contains high buildings, 
very low vegetation and medium houses as an urban environment. Moreover, Weissberger model is able to estimate the path loss due to the presence of one or more trees in a point-to-point telecommunication link.

\subsection{Weissberger's Model}

Weissberger's model, is a radio wave propagation model that estimates the path loss due to the presence of one or more trees in a point-to-point telecommunication link. This model is more applicable when there is an obstruction made by some foliage in the link between the transmitter and receiver. Weissberger's model is calculated as [20]:

$$
\begin{array}{ll}
L=1.33 f^{0.284} d^{0.588} & , 14<d \leq 400 \\
L=0.45 f^{0.284} d & , 0<d \leq 14
\end{array}
$$

where $d$ is the depth of foliage through the transmission path in meters; $L$ is the loss due to the foliage in $\mathrm{dB} ; f$ is the transmission frequency in $\mathrm{GHz}$.

\subsection{COST 231 Walfish-Ikegami (W-I) Model}

COST 231 (W-I) model is considered as the most appropriate model for rural and suburban environments which have regular building height. Moreover, this model gives more accurate path loss prediction. It identifies various terrain with different parameters. The description of the model for NLOS condition is expressed as the following equations [21]:

$$
\begin{gathered}
P L_{N L O S}=L_{F S L}+L_{r t s}+L_{m s d} \text { for suburban \& urban } \\
P L_{N L O S}=L_{F S L} \quad \text { when } L_{r t s}+L_{m s d}>0
\end{gathered}
$$

where $L_{F S L}$ is Free space loss; $L_{r t s}$ is roof top to street diffraction; $L_{m s d}$ is multi screen diffraction loss.

Moreover, the Free space loss equation is calculated as:

$$
L_{F S L}=32.45+20 \log (d)+20 \log (f)
$$

The roof top to street diffraction is computed as, [18]:

$$
\begin{array}{cc}
L_{r t s}=-10 \log (\mathrm{w})-16.9+10 \log (\mathrm{f})+20 \log \left(H_{\text {mobile }}\right)+L_{\text {ori }} \\
L_{r t s}=0 \quad \text { if } h_{\text {roof }}>h_{\text {mobile }} \\
\text { where } \\
L_{\text {ori }}=2,5+0,075(\varphi-35), & \text { for } 35 \leq \varphi \leq 55 \\
L_{\text {ori }}=4-0,114(\varphi-55), & \text { for } 55 \leq \varphi \leq 90 \\
L_{\text {ori }}=-10+0,354 \varphi, & \text { for } 55 \leq \varphi \leq 90
\end{array}
$$


We have to observe that:

$\Delta h_{\text {base }}=h_{\text {base }}-h_{\text {roof }}$

$\Delta h_{\text {mobile }}=h_{\text {roof }}-h_{\text {mobile }}$

The $L_{m s d}$ (multi screen diffraction loss) is calculated as:

$L_{m s d}=k_{a}+L_{b s h}+k_{d} \log _{10}(d)+k_{f} \log _{10}(f)-9 \log _{10}(B)-9 \log _{10}(f)$ for $L_{m s d}>0$

$L_{m s d}=0 \quad, \quad$ for $L_{m s d}<0$

Where

$L_{b s h}=0 \quad, \quad$ for $h_{\text {base }} \leq h_{\text {roof }}$

$L_{b s h}=-18 \log _{10}\left(1+\Delta h_{\text {base }}\right)$, for $h_{\text {base }}>h_{\text {roof }}$

$k_{d}=18+15\left(\frac{\Delta h_{\text {base }}}{h_{\text {roof }}}\right)$, for $h_{\text {base }} \leq h_{\text {roof }}$

$k_{d}=18$

, for $h_{\text {base }}>h_{\text {roof }}$

$k_{a}=54-0.8 \Delta h_{\text {base }} \quad$, for $d \geq 0,5 \mathrm{~km}$ and $h_{\text {base }} \leq h_{\text {roof }}$

$k_{a}=54-0.8 \Delta h_{\text {base }}\left(\frac{d}{0.5}\right)$, for $\mathrm{d}<0.5 \mathrm{~km}$ and $h_{\text {base }} \leq h_{\text {roof }}$

$k_{a}=54 \quad$, for $h_{\text {base }}>h_{\text {roof }}$

$k_{f}=-4+1.5\left(\frac{f}{925}-1\right)$, for urban areas

$k_{f}=-4+0.7\left(\frac{f}{925}-1\right)$, for suburban areas

where $\mathrm{B}$ is the building to building distance in meters; $d$ is the distance between transmitter and receiver antenna in meters; $f$ is the frequency in $\mathrm{GHz} ; \varphi$ is the street orientation angle degree; $w$ is the street width in meters.

Also, the equation for LOS condition is expressed as:

$$
P L_{\text {los }}=20 \log (f)+42,6+26 \log (d)
$$

\section{Experimental Method}




\subsection{Measurement Setup and Apparatus}

In this paper, measured data was analyzed and compared with the simulated data of Weissberger and COST $231 \mathrm{~W}$-I models in urban area. Measured data was analyzed and compared with the theoretical and simulated data of Weissberger and COST $231 \mathrm{~W}$-I models in urban area. In addition, a comparative analysis is performed with the predictions that provided by the existing empirical models, namely Weissberger and COST 231 Walfish-Ikegami (W-I) models.

The measured data was implemented at the frequency of $900 \mathrm{MHz}$. It was preferable during the measurement setup to use the frequency band of $900 \mathrm{MHz}$ as the properties of waves have good match with coverage area in such urban environment in order to investigate the behavior of the signal attenuation and propagation slop. Furthermore, the measurements could be susceptible for guided waves propagation phenomenon as the street orientation and structure which can help the radio waves to propagate smoothly. Thus, one of the main goals of this research is to investigate which factors must be tuned to perform an optimal design of a wireless communication system at the $900 \mathrm{MHz}$ band to develop dedicated planning methodologies for wireless communication deployment. Therefore, for this purpose, it is crucial to acquire knowledge of radio propagation characteristics at the $900 \mathrm{MHz}$ band. Measurement locations included both Line-Of-sight (LOS) and Non Line-Of-Sight (NLOS) propagation conditions. A comparative analysis is performed with the predictions that provided by the existing empirical models, namely Weissberger and COST 231 Walfish-Ikegami (W-I) models. The measurements are collected and analysed to derive the path loss behavior at $900 \mathrm{MHz}$. The values of the cable loss, insertion losses and antenna gain were removed to estimate the path attenuation only due to propagation environment. The mobile receiver comprises a suitable band pass filters and a low noise amplifier connected to a spectrum analyzer RohdeSchwarz FSH which is a portable spectrum analyzer ranging from $100 \mathrm{kHz}$ up to $3.6 \mathrm{GHz}$ with two-port network analyzer as shown in Fig. 1. A narrowband continuous wave signal with the carrier frequency of $900 \mathrm{MHz}$ was fed to transmitter antenna with $40 \mathrm{dBm}$ power. The receiver was recording approximately 320 samples for every meter from the travelling distance to record the large average values and the transmitter height was about 1.5 meters. For each measure, test run had duration of 3 minutes. The measurements are collected and analysed to derive the path loss behavior at $900 \mathrm{MHz}$. The mobile receiver comprises a suitable band pass filters and a low noise amplifier connected to a spectrum analyzer RohdeSchwarz FSH which is a portable spectrum analyzer ranging from $100 \mathrm{kHz}$ up to $3.6 \mathrm{GHz}$. So, a spectrum analyzer was used to record the channel response. The mean analysis of all the samples was performed to validate the collected data for each measure. Thus, some measures were excluded whose samples were considered very far from the plurality of the values in order to remove the measurement noise that caused by some obstacles like buses, foliage, cars and trees. 


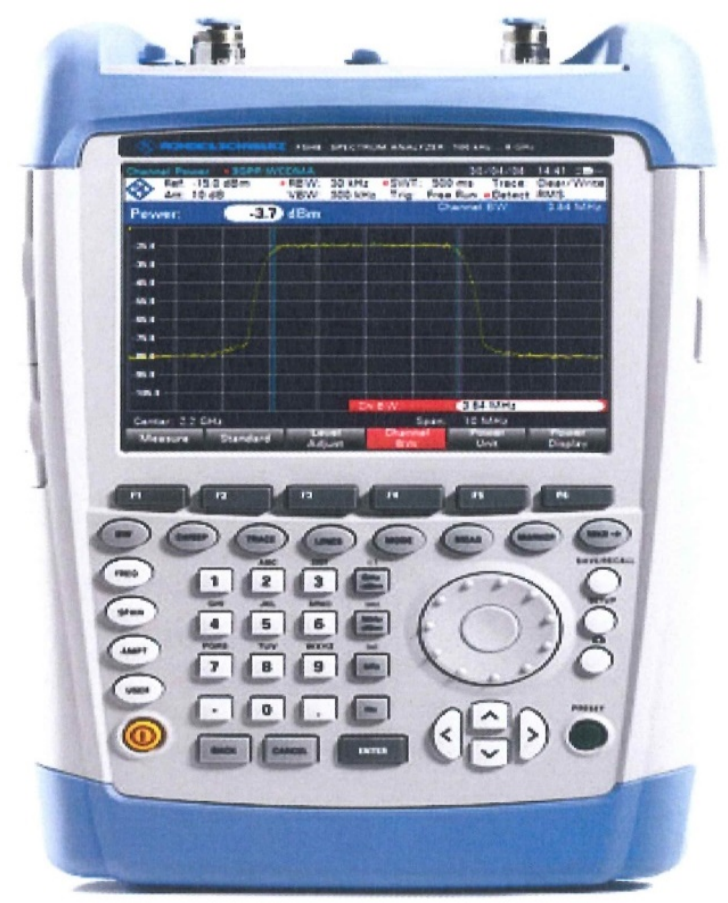

Fig. 1. Portable Spectrum Analyzer up to 3,6 GHz

\subsection{Measurement Environment}

The measurements survey was conducted at the campus of Cairo University in Dokki region which is a district in Giza city, which is part of greater Cairo in Egypt. Dokki is situated on the western bank of the Nile directly across from Downtown Cairo. Measurements were performed during 2015 at the campus of Cairo University in Dokki region which reflecting a typical urban area with high buildings, very low vegetation, medium houses and vast streets. Dokki region was chosen to introduce a typical urban region that consists of densely buildings and concrete and brick are the most commonly used building materials. Moreover, roads are predominantly wide and sufficiently rectilinear. The average building height is approximately more than 16 meters and buildings may have five to eight floors. In some narrow streets which consist of medium buildings heights, the average building height is more than 14 meters and buildings may have four to six floors.

\subsection{Measurement Procedure}

The measurements were conducted at the frequency of $900 \mathrm{MHz}$ frequency band using a continuous wave mode transmitter and the stationary receiver was placed in a moving vehicle. The simulation of the selected models was performed by using MATLB R2014b. The simulation process flow chart that was used for the simulation of the selected models is described in Fig. 2.

It was observed that the average simulation time for each run was about 49 sec during the simulation process of each channel model. The path loss of channel models was calculated by 
setting the mobile transmitter power equal to $35 \mathrm{dBm}$ and base station transmitter power equal to $43 \mathrm{dBm}$. The simplified experimental setup block diagram is shown in Fig. 3. Related to the parameters of COST $231 \mathrm{~W}$-I model; the street width was set to be $25 \mathrm{~m}$ and street orientation angel equal to 30 degree. Extensive measurements are carried out over distances ranging from $130 \mathrm{~m}$ to approximately $1.8 \mathrm{Km}$ from the transmitter.

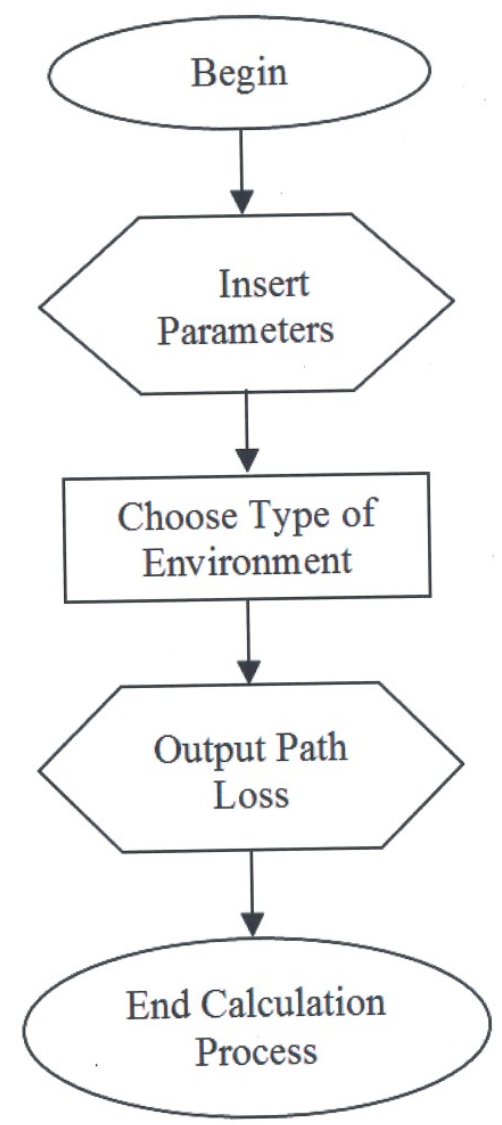

Fig. 2. Simulation process flow chart

Transmitter

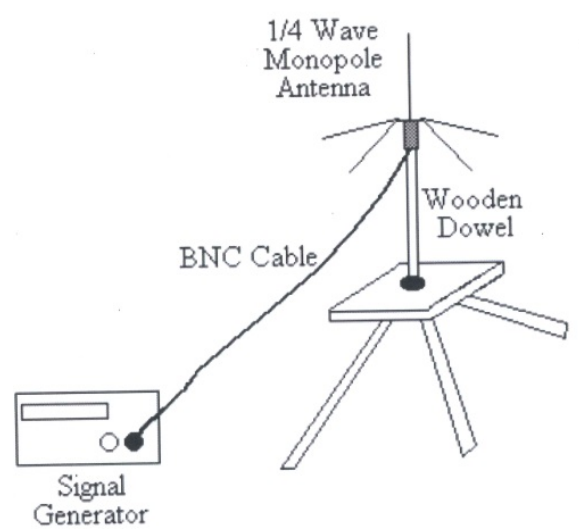

Receiver

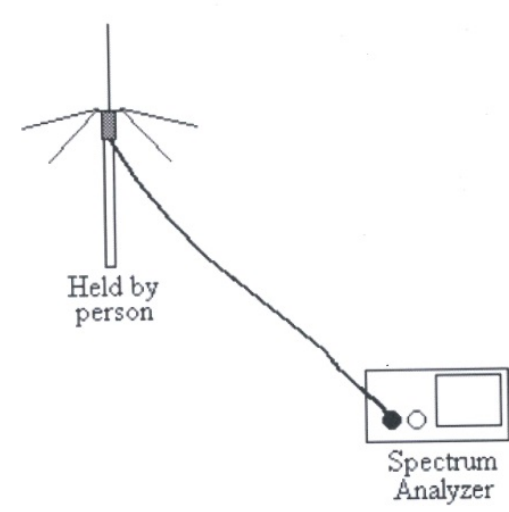

Fig. 3. Experimental setup block diagram 


\section{Results and Analysis}

In this section, the path loss behaviour derived from our measurement campaign is compared with the predictions made by two of the empirical models, namely Weissberger and COST 231 Walfish-Ikegami (W-I) models. Thus, these models were described in terms of their parameters and characteristic equations. The path loss versus the distance for LOS and NLOS measures are shown in Fig. 4 and Fig. 5, respectively.

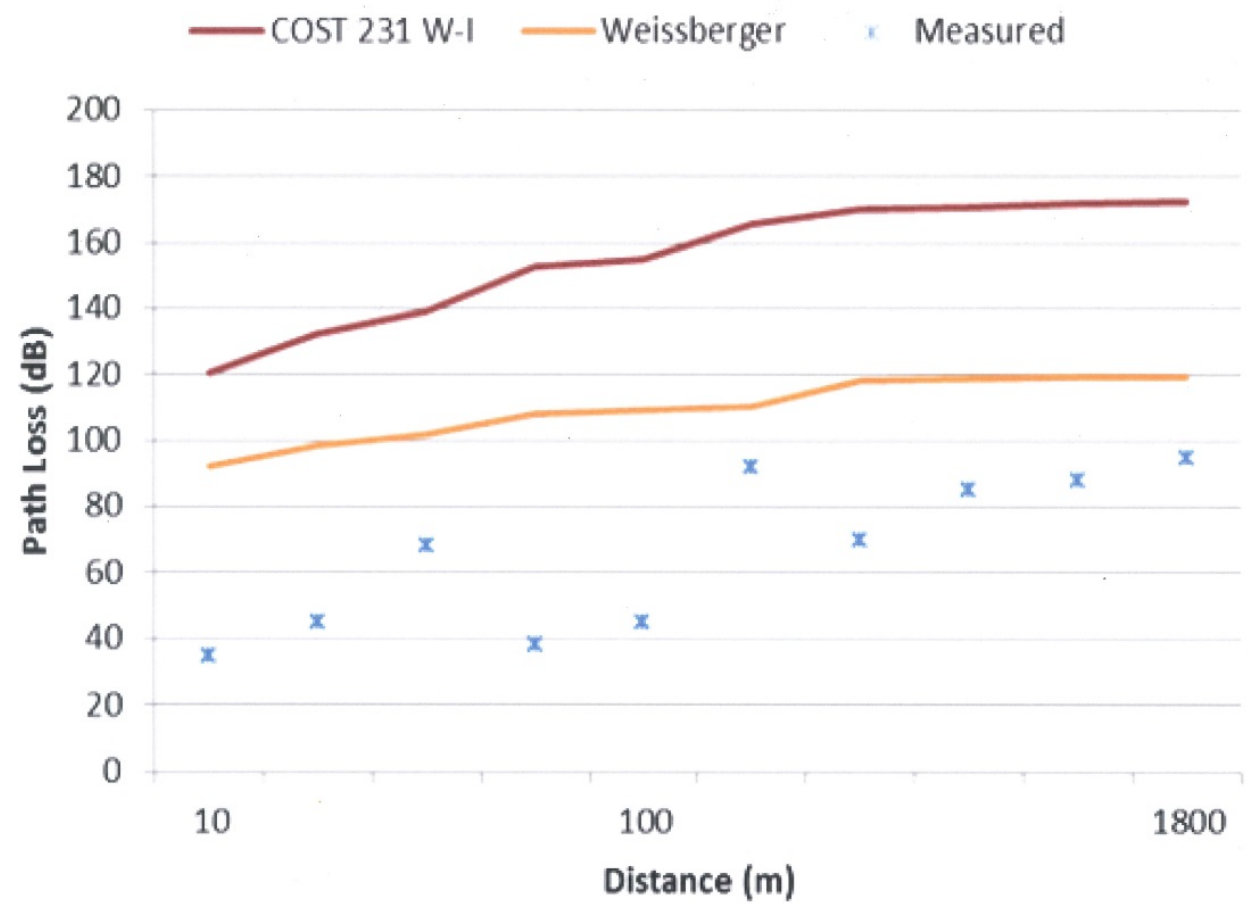

Fig. 4. Measured path loss in LOS condition with empirical models.

By taking into account the results of COST $231 \mathrm{~W}$-I model, it was noted that these results are in good agreement with the results of the same model which described in [22]. Also, attention should be paid for the results in [23] which stated that two different types of cells deployment which are random building environment and high urban environment with tall buildings have been compared and studied. Advanced network model for Device-to-Device heterogeneous network was developed based on the Poisson point process combined with K-means clustering method which is able to reflect the random user devices. Stochastic geometry models provide good understanding of the mobile network behavior. Using PPP and HCPP processes reflects positions of base stations with good approximation, while Voronoi tessellation provides well tractable and feasible reflection of signal propagation areas. That paper stated that optimal network planning is a significant challenge for the future heterogeneous networks with high number of small cells. Simulation results show that heterogeneous network provides significantly higher performance in terms of throughput and signal-to-interference-plus-noise ratio distribution. 
In order to quantitatively estimate the appropriateness of the described empirical models, we compared the predicted propagation loss by both of Weissberger and COST $231 \mathrm{~W}$-I models with the measurements and the appreciation is performed for LOS and NLOS cases. We have to bear in mind that in urban areas the path loss estimation was influenced according to the greater distances. In the beginning of the measurement in NLOS condition $23 \mathrm{~dB}$ loss occurred after 18 meters from the measurement survey. It should be noted that the resulting overestimation of the propagation path loss impacts the prediction of the service. Thus, the above discussion highlights the relevance of a precise estimation of the propagation loss. Results in [24] presented a new deterministic approach for analysis of multi-tier heterogeneous network that was proposed in the research based on the fractal geometry. Also, two models were proposed for regular and non-regular environment. Proposed solution is well tractable and suitable for every possible topology of heterogeneous network, providing exact information about network capacity and co-channel interference. Two fractal models were developed based on triangular and rectangular pattern and basic geometric equations were derived for multi-tier network topology. Frequency reuse was analyzed for cellular and backhaul spectrum. Results of simulations stated that proposed fractal modeling provides more accurate network analysis in terms of capacity and interference influence in multi-tier heterogeneous networks.

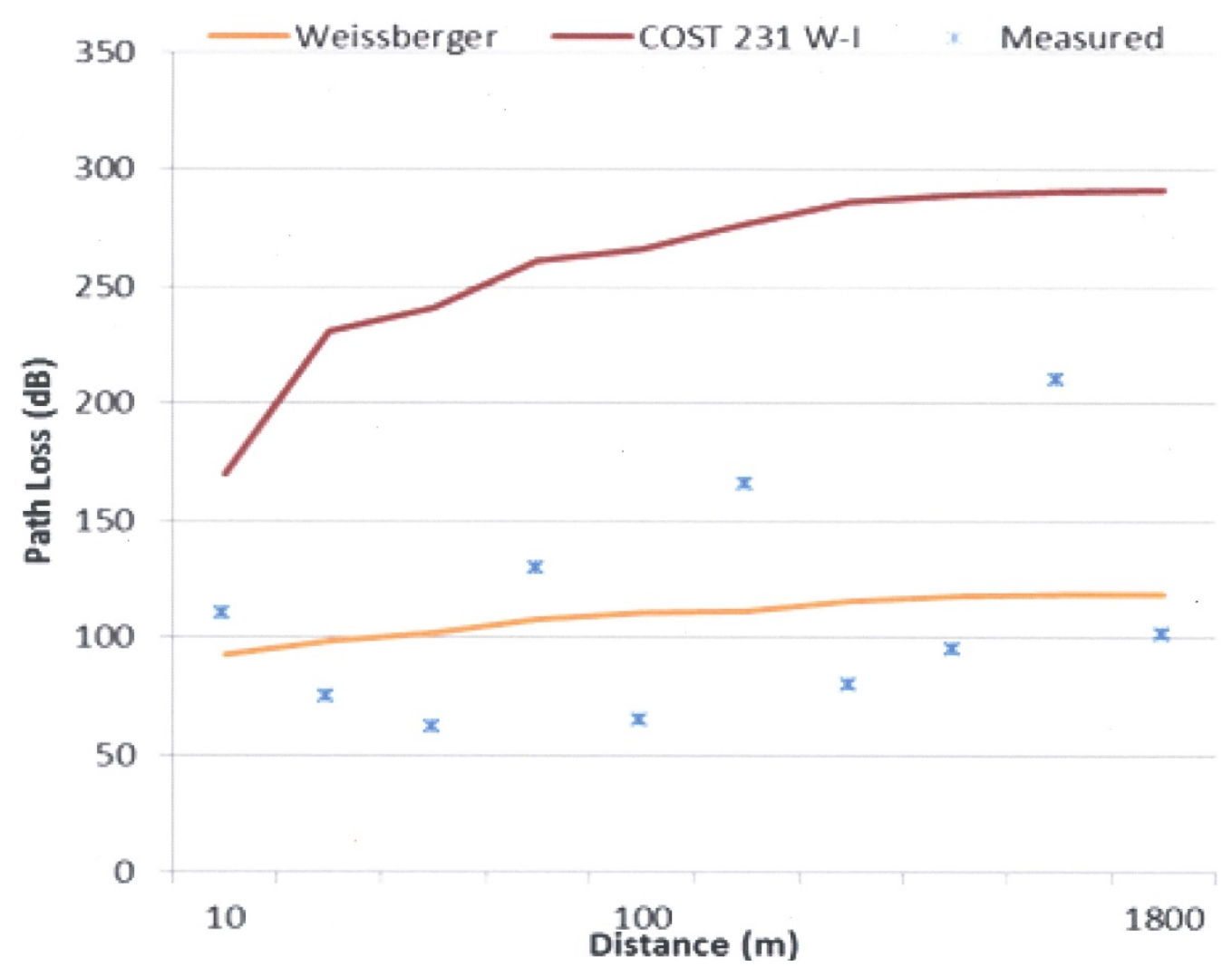

Fig. 5. Measured path loss in NLOS condition with empirical models.

The measured path loss values were little bit different compared to the path loss values that stated in [25] as an urban environment. Moreover, Fig. 6 shows the average analysis of path 
loss values of the selected models. Also, it was obvious that at short distances from the transmitter the path loss decreases according to the increment of the transmitter height. Thus, the height and position of the transmitter, broken connectors and bad connected cables affect path loss estimation.

Based on the above discussion it was observed during the practical measure that 32 meters was approximately precise as an assumption for the break point distance. It was noted that the predicted path loss values of Weissberger model are close to the experimented values in LOS condition. Moreover, the measurements could be susceptible for guided waves propagation phenomenon as the street orientation and structure can help the radio waves to propagate smoothly. Moreover, results in [26] described a new resource allocation method for multi-tier HetNet based on the fractal geometry that was proposed and frequency reuse was analyzed in order to assess interference influences between co-channel small cells. SINR optimization function has been derived and both of the two cases underutilization and overloading have been studied. Finally, we have to point out that the channel modeling is necessary for characterizing the impulse response and evaluate the path loss of a propagation channel.

As a conclusion to be drawn from the above discussion, it was observed that the differences between the measured values and the empirical models were due to existence of obstruction obstacles, reflections and scatters. Previous studies observed that propagation beyond corners of the buildings can be the reason of 29-33 dB attenuation of path loss values in urban area. Also, lower receiver heights cause higher path loss that is more authoritative at less distance from the transmitter.

From the analyzed results of Weissberger and COST $231 \mathrm{~W}$-I models it should be noticed that Weissberger model is more appropriate in the case of LOS than NLOS at the urban area. Therefore, we may compare another type of channel propagation model with Weissberger model for the both cases LOS and NLOS at urban environment.

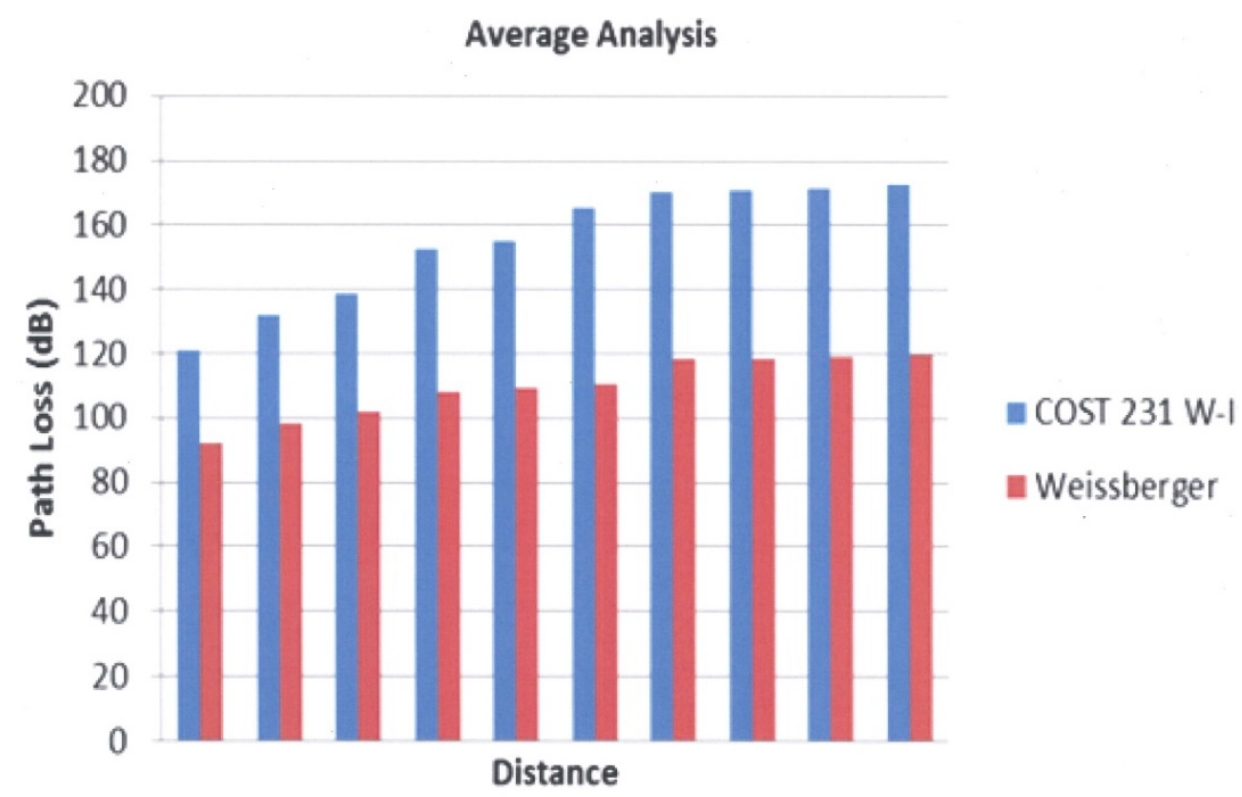

Fig. 6. Average analysis of path loss for COST 231 W-I and Weissberger models. 
As a conclusion in the case examined here, it can be realized that the analysis of simulations and measurements focus on predicting the average signal strength at the receiver at a given distance from the transmitter as well as the variability of the signal strength in a specific environment. Moreover, these measurements will provide a basis for defining planning methodologies that will be a matter of further research.

\section{Conclusion and Future Work}

This research presents a comparison between the measured and simulated data of the path loss at the campus of Cairo University in Dokki region by considering both of LOS and NLOS conditions. An extensive measurement campaign is performed at $900 \mathrm{MHz}$ frequency band. The comparison between our results and the predictions which obtained by applying suitable empirical models shows that the existing models can be inadequate in urban scenarios as they could lead to overestimate the propagation loss. It is obvious that high density systems suffer from performance reduce due to the intervention that could be underevaluated through network planning when an over estimation of the path loss happens. Therefore, it is paramount to evaluate an accurate propagation loss to provide the network designers pragmatic radio parameters to achieve an acceptable level of quality of service for the users. In the near future, we plan to compare the measurements from suburban area with both of Weissberger and COST $231 \mathrm{~W}$-I models. Then, the obtained results should be analyzed especially with COST $231 \mathrm{~W}$-I model in order to find out which model is better in urban environment and which one is better in suburban environment. So, we may try to find appropriate parameters for COST $231 \mathrm{~W}-\mathrm{I}$ model which could be suitable for all types of terrain.

\section{ACKNOWLEDGMENT}

This research is supported by the project SP 2016/143, "Research of antenna systems; effectiveness and diagnostics of electric drives with harmonic power; reliability of the supply of electric traction; issue data anomalies".

Also, the authors would like to express their thanks to all the members of Department of Electrical Engineering at VSB - Technical University of Ostrava for their valuable instructions and support.

\section{References}

[1] Rappaport, T.S., Wireless Communications, Prentice-Hall, Upper Saddle River, New Jersey, Ch. 3 \& 4, 1996. Article (CrossRef Link)

[2] Chu, M.J. \& Stark, W.E., "Effect of mobile velocity on communications in fading channels,” IEEE Trans. on Veh. Techn 49(1): 202-210, 2000. Article (CrossRef Link)

[3] Linnartz, J.P., “Narrowband Land-Mobile Radio Networks,” Artech house, 1993. Article (CrossRef Link).

[4] Cox et al., “800MHz Attenuation measured in and around suburban houses,” Bell Laboratory Technical Journal 673(6): 921-954, 1984. Article (CrossRef Link).

[5] Laitinen, E. et al., “Access point significance Measures in WLAN-based location,” in Proc. of the 9th Workshop on Positioning, Navigation and Communication (WPNC), 2012.

Article (CrossRef Link). 
[6] Honcharenko, W. \& Bertoni, H. L., "Transmission and reflection characteristics at concrete block walls in the UHF band proposed for future PCS,” IEEE Trans. Antennas Propagation 42: 232-239, 1994. Article (CrossRef Link).

[7] Obot, A et al., “Comparative Analysis of Pathloss Prediction Models for Urban Microcellular," Nigerian Journal of Technology 30(3): 50-59, 2011. Article (CrossRef Link).

[8] Bi, Q et al., "Wireless mobile communications at the start of the 21st century," IEEE Commun. Mag 39: 110-116, 2001. Article (CrossRef Link).

[9] Oeting, J., “Cellular Mobile Radio - An Emerging Techonology," IEEE Communications Magazine: 10-15, 1983. Article (CrossRef Link).

[10] Keenan, J.M. \& Motley, AJ., "Radio coverage in buildings,” Er. Telerom. Technol. J., 8, (l): 19-24, 1990. Article (CrossRef Link).

[11] Seidel, S.Y. \& Rappaport, T.S., “914 MHz path loss prediction models for indoor wireless communications in multifloored buidlings," IEEE Trans. Antennas \& Propagation, AP-40, (2): 207-217, 1992. Article (CrossRef Link).

[12] Hashemi, H., “Impulse response modelling of indoor radio propagation channels,” IEEE J. Sel. Areas Commun, SAC-1 1: 1788-1796, 1993. Article (CrossRef Link).

[13] Rizk, K et al., Lamppost and panel scattering compared to building reflection and diffraction in COST 259 TD, Turin, Italy: 158-167, 1993. Article (CrossRef Link).

[14] Llacer, L. Juan \& Cardona, N., "UTD solution for the multiple building diffraction attenuation function for mobile radiowave propagation,” Electron. Lett 33(1): 92-93, 1997. Article (CrossRef Link).

[15] Erceg, V et al., "An empirically based path loss model for wireless channels in suburban environments," IEEE Journal on Selected Areas of Communications 17: 1205-1211, 1999. Article (CrossRef Link).

[16] Saunders R.S et al., "Making Wireless Networks deliver," Ingenia of the Royal Academy of Engineering (12): pp 36-40, 2002. Article (CrossRef Link).

[17] Satvir Singh Sidhu et al., "Implementation of 3-D Ray Tracing Propagation Model for Indoor Wireless Communication,” International Journal of Electronics Engineering 4 (1): 43- 47, 2012. Article (CrossRef Link).

[18] Isabona, J et al., "Radio Field Strength Propagation Data and Pathloss calculation Methods in UMTS Network,” Advances in Physics Theories and Applications, 21: 54-68, 2013. Article (CrossRef Link).

[19] Samimi, M. K \& Rappaport T. S., “3-D Statistical Channel Model for Millimeter-Wave Outdoor Mobile Broadband Communications, in Proc. of 2015 IEEE International Conference on Communications (ICC), 2015. Article (CrossRef Link).

[20] Seybold, J., Introduction to RF Propagation. John Wiley and Sons, 2005. Article (CrossRef Link).

[21] Alam, Md et al., "Performance Evaluation of Different Frequency Bands of WiMAX and Their Selection Procedure,” International Journal of Advanced Science and Technology 62: 1-18, 2014. Article (CrossRef Link).

[22] Jadhav, A.N. \& Kale, S., "Suburban Area Path loss Propagation Prediction and Optimisation Using Hata Model at 2375 MHz,” International Journal of Advanced Research in Computer and Communication Engineering 3(1), 2014. Article (CrossRef Link).

[23] Maksymyuk, T et al., "Stochastic Geometry Models for 5G Heterogeneous Mobile Networks," Smart Computing Review, vol. 5, no. 2, pp. 89-101, 2015. Article (CrossRef Link).

[24] Maksymyuk, T et al., "Fractal Modeling for Multi-Tier Heterogeneous Networks with Ultra-High Capacity Demands,” Smart Computing Review, vol. 5, no. 3, pp. 346-355, 2015. Article (CrossRef Link).

[25] Sun, S et al., "Propagation Path Loss Models for 5G Urban Microand Macro-Cellular Scenarios," in Proc. of 2016 IEEE 83rd Vehicular Technology Conference (VTC2016-Spring), 2016.

Article (CrossRef Link). 
[26] Maksymyuk, T et al., "Fractal geometry based resource allocation for 5G heterogeneous networks," in Proc. of IEEE Second International Scientific-Practical Conference on Problems of Info communications Science and Technology (PIC S\&T), pp. 69-72, 2016.

Article (CrossRef Link).

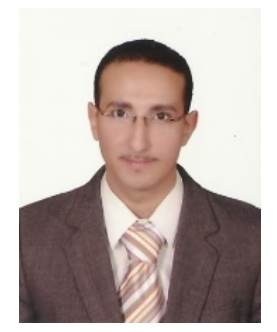

Yahia ZAKARIA from the Faculty of Electrical Engineering and Computer Science, VSB-Technical University of Ostrava, Czech Republic. He is a permanent member of the research corporation at the Engineering Research Division, National Research Centre (NRC), Ministry of Scientific Research, Cairo, Egypt. In addition, he was one of the members of the Technical Office for follow-up and evaluation of performance at the National Research Centre since 2008 until 2010. He participated in many seminars and lectures at the German Academic Exchange Service (DAAD), Germany. He is a reviewer in three International Scientific Journals. He joined the department of Electronic and Electrical Engineering, University of Strathclyde, United Kingdom, UK as practical research traineeship in December 19, 2016. His current research interests include Cellular Communication Systems, Broadband Networks, Mobile Communications, Robotics, Security for Future Networks, Channel propagation Models of Mobile Communication, Network Engineering, RF Signals, Wireless Communication Technologies, Cloud Computing and LTE Technology for Wireless Communication Systems.

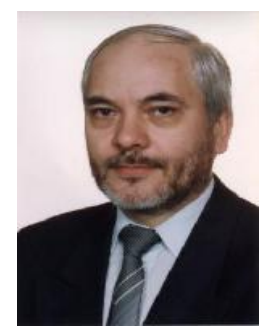

Lubomír IVÁNEK is an Associate Professor in the VSB-Technical University of Ostrava, Faculty of Electrical Engineering and Computer Science, Czech Republic. He graduated from the VSB-Technical University Ostrava - Faculty of Mechanical and Electrical Engineering, earned his Ph.D. degree from the Czech Technical University in Prague, Department of Theoretical Electrical Engineering. Associate Professor degree received at the Technical University of Ostrava in the field of Theoretical Electrical Engineering. His research involves the waves propagation and antennas, mathematical modelling of the electromagnetic field, stray current under electric tractions.

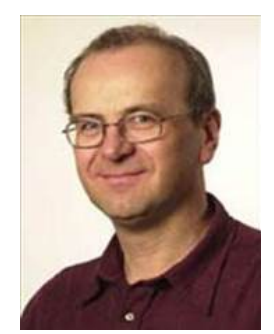

Ivan GLESK joined the Department of Electrical Engineering at Princeton University, USA in 1991 where he became Senior Research Scholar and Manager of the Lightwave Communication Research Laboratory. In 2007, he joined Electronic and Electrical Engineering Department at University of Strathclyde, UK as Professor of Broadband Communication Systems. Current research interests: Broadband, OFDM communication systems, physical layer security, applications of optical amplifiers and short pulse and Channel propagation Models of Mobile Communication. 